\title{
Heat Exchange Between Electrons and Phonons in Nanosystems at Sub-Kelvin Temperatures
}

\author{
Dragoş-Victor Anghel ${ }^{1, \star}$ and Sergiu Cojocaru ${ }^{1, \star \star}$ \\ ${ }^{1}$ Institutul National de C\&D pentru Fizica si Inginerie Nucleara - Horia Hulubei, 077125 Magurele, Romania
}

\begin{abstract}
Ultra-sensitive nanoscopic detectors for electromagnetic radiation consist of thin metallic films deposited on dielectric membranes. The metallic films, of thickness $d$ of the order of $10 \mathrm{~nm}$, form the thermal sensing element (TSE), which absorbs the incident radiation and measures its power flux or the energies of individual photons. To achieve the sensitivity required for astronomical observations, the TSE works at temperatures of the order of $0.1 \mathrm{~K}$. The dielectric membranes are used as support and for thermal insulation of the TSE and are of thickness $L-d$ of the order of $100 \mathrm{~nm}$ ( $L$ being the total thickness of the system). In such conditions, the phonon gas in the detector assumes a quasi-two-dimensional distribution, whereas quantization of the electrons wavenumbers in the direction perpendicular to the film surfaces leads to the formation of quasi twodimensional electronic sub-bands. The heat exchange between electrons and phonons has an important contribution to the performance of the device and is dominated by the interaction between the electrons and the antisymmetric acoustic phonons.
\end{abstract}

High sensitivity electromagnetic radiation detectors for space applications are nanometer-size devices which work at sub-Kelvin temperatures [1,2]. Such a detector consists of a thermal sensing element (TSE) which is deposited on a supporting membrane. The TSE is formed of one or several metallic layers and has the role of absorbing and measuring the incident electromagnetic radiation. The supporting membrane is dielectric and provides the thermal insulation of the TSE from the bulk material [1-4]. To ensure the level of sensitivity required by the astrophysical observations, the thickness of the TSE should be of the order of $10 \mathrm{~nm}$, the thickness of the supporting membrane is of the order of $100 \mathrm{~nm}$, whereas the working temperature of the device may be about $100 \mathrm{mK}[5,6]$. The layers of materials that form the detector are shown schematically in Fig. 1.

The TSE may consist of a normal metal strip coupled to a superconducting antenna [2, 3, 5-9]. When the incident radiation is absorbed, its energy is dissipated into the normal metal strip. At the detector's working temperature, the electrons are weakly coupled to the lattice and therefore we can associate an effective temperature to each of these subsystems: $T_{e}$ will denote the temperature of the electrons and $T_{p h}$ will denote the temperature of the lattice (phonons).

Being at different temperatures, a net heat power $P$ flows between the electron system and the phonon system, due to the electron-phonon interaction. This interaction is described by the deformation potential Hamiltonian [10-12]

$$
\hat{H}_{d e f}=\frac{2}{3} E_{F} \int_{V_{e l}=A \times d} d^{3} \mathbf{r} \Psi^{\dagger}(\mathbf{r}) \Psi(\mathbf{r}) \nabla \cdot \mathbf{u}(\mathbf{r}),
$$

\footnotetext{
^e-mail: dragos@theory.nipne.ro

${ }^{\star}$ e-mail: scojocaru@ theory.nipne.ro
} 


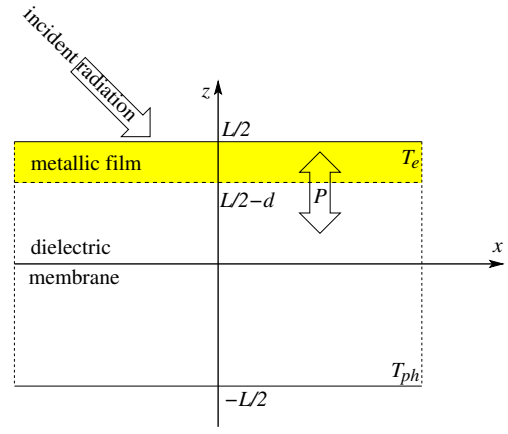

Figure 1. The schematic representation of the metallic film (the layer between $z=L / 2-d$ and $L / 2$ ), on top of the supporting dielectric membrane (between $z=-L / 2$ and $x=L / 2-d$ ). The incident radiation is absorbed by the metallic film, increasing the temperature of the electrons $T_{e}$ above the temperature of the lattice $T_{p h}$. The electron-phonon heat exchange is $P$. The upper and lower surfaces of the film and membrane are parallel to the $(x y)$ plane.

where $E_{F}$ is the Fermi energy of the electrons, $A$ is the area of the metallic film, $d$ is the thickness (see Fig. 1), $\Psi^{\dagger}(\mathbf{r})$ and $\Psi(\mathbf{r})$ are the electron field creation and annihilation operators, respectively, whereas $\mathbf{u}$ is the phonon field. The volume of the metallic film is $V_{e l} \equiv A d$ and we do not take into account any other interaction in the system (like, for example, electron-electron, phonon-phonon or interaction with impurities). For three-dimensional (3D) systems, the heat power $P^{3 D} \propto T_{e}^{5}-T_{p h}^{5}[13]$. If the phonons are confined to a lower dimensionality $s$, then $[12,14,15]$

$$
P^{s D} \propto T_{e}^{s+2}-T_{p h}^{s+2} .
$$

In general, we write $P^{s D} \equiv P^{(0)}-P^{(1)}$, where $P^{(0)}$ is defined as the heat power from electrons to phonons and $P^{(1)}$ is the heat power from phonon to electrons, so that, from Eq. (2), we have $P^{(0)} \propto T_{e}^{s+2}$ and $P^{(1)} \propto T_{p h}^{s+2}$.

In the detector systems investigated by us, the supporting membrane is made of silicon nitride $\left(\mathrm{SiN}_{x}\right)$ and the metallic film is copper $(\mathrm{Cu})$. The phonon modes and the thermal properties of nanoscopic $\mathrm{SiN}_{x}$ membranes have been investigated, for example, in [16-18] and it was noticed that in a temperature range below $1 \mathrm{~K}$ a dimensionality crossover of the phonon modes takes place, namely a quasi-two-dimensional (2D) phonon gas distribution transforms - as the temperature increases - into a 3D distribution. For example, the phonon gas in a $100 \mathrm{~nm}$ thick membrane is well approximated by a $2 \mathrm{D}$ distribution at temperatures below $200 \mathrm{mK}$ and therefore one would expect $s=2$ and the power flux proportional to $T_{e}^{4}-T_{p h}^{4}$ in this temperature range. If one takes into account the discretization of the electrons' wavevectors in the direction perpendicular to the surface of the metallic layer (we shall call this the quantum well description-QW), together with the confinement of the phonon modes, the form (2) changes. The quasi-2D phonon modes in the system can be divided into three categories [19]: horizontal shear $(h)$, symmetric $(s)$ and antisymmetric $(a)$ modes. From these three types of phonons, only the $s$ and $a$ modes interact with the electrons and therefore contribute to the heat flux. The heat flux due to the $s$ modes is $P_{Q W s}^{2 D} \propto T_{e}^{4}-T_{p h}^{4}$ and the heat flux due to the $a$ modes is $P_{Q W a}^{2 D} \propto T_{e}^{3.5}-T_{p h}^{3.5}$; the total flux is $P_{Q W}^{2 D}=P_{Q W s}^{2 D}+P_{Q W a}^{2 D}$. In the low temperature limit $\left(T_{e}\right.$ and $T_{p h} \rightarrow 0$ ), the antisymmetric modes have the dominant contribution to the heat exchange, as can be seen in Fig. 2.

In conclusion, the heat exchange between electrons and phonons in the layered structure investigated is dominated by the interaction between the electrons and the antisymmetric phonon modes, which leads to a temperature dependence $P_{Q W}^{2 D} \propto T_{e}^{3.5}-T_{p h}^{3.5}$ that does not support the conjecture (2).

\section{Acknowledgement}

Financial support from the Romnian ANCS project PN 16420101 and from the Romania-JINR collaboration projects, JINR Order 220/10.04.2017, positions 18, 19, 21, 22 are gratefully acknowledged. 


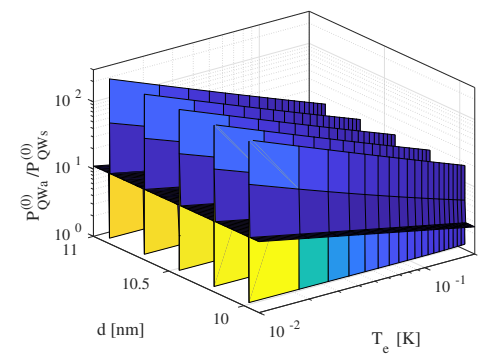

Figure 2. The ratios $P_{Q W a}^{(0)} / P_{Q W s}^{(0)}$ for $L=100 \mathrm{~nm}$. In the temperature range of interest $\left(T_{e}<0.2 \mathrm{~K}\right)$, the dominant contribution comes from the antisymmetric phonons. The same is true for $P_{Q W a}^{(1)} / P_{Q W s}^{(1)}$. The sharp crests that appear parallel to the $T_{e}$ axis are exceptions to the simple power-law dependence of $P_{Q W}^{2 D}$ and are not discussed here (see [11] for details).

\section{References}

[1] M.J. Myers, W. Holzapfel, A.T. Lee, R. O'Brient, P.L. Richards, H.T. Tran, P. Ade, G. Engargiola, A. Smith, H. Spieler, Appl. Phys. Lett. 86, 114103 (2005)

[2] F. Giazotto, T.T. Heikkilä, A. Luukanen, A.M. Savin, J.P. Pekola, Rev. Mod. Phys. 78, 217 (2006)

[3] D.V. Anghel, L. Kuzmin, Appl. Phys. Lett. 82, 293 (2003)

[4] K.L. Denis, N.T. Cao, D.T. Chuss, J. Eimer, J.R. Hinderks, W. Hsieh, S.H. Moseley, T.R. Stevenson, D.J. Talley, K. U.-yen et al., AIP Conf. Proc. 1185, 371 (2009)

[5] D.V. Anghel, A. Luukanen, J.P. Pekola, Appl. Phys. Lett. 78, 556 (2001)

[6] D.V. Anghel, J.P. Pekola, J. Low Temp. Phys. 123, 197 (2001)

[7] M. Nahum, J.M. Martinis, Appl. Phys. Lett. 63, 3075 (1993)

[8] M. Nahum, P.L. Richards, C.A. Mears, IEEE Trans. Appl. Superc. 3, 2124 (1993)

[9] L.S. Kuzmin, Physica B p. 2129 (2000)

[10] J.M. Ziman, Electrons and Phonons (Harcourt College Publishers, 1976) 566 pp.

[11] D. Anghel, S. Cojocaru, Solid State Commun. 227, 56 (2016)

[12] S. Cojocaru, D.V. Anghel, Phys. Rev. B 93, 115405 (2016)

[13] F.C. Wellstood, C. Urbina, J. Clarke, Phys. Rev. B 49, 5942 (1994)

[14] J.K. Viljas, T.T. Heikkilä, Phys. Rev. B 81, 245404 (2010)

[15] F.W.J. Hekking, A.O. Niskanen, J.P. Pekola, Phys. Rev. B 77, 033401 (2008)

[16] D.V. Anghel, J.P. Pekola, M.M. Leivo, J.K. Suoknuuti, M. Manninen, Phys. Rev. Lett. 81, 2958 (1998)

[17] T. Kühn, D.V. Anghel, J.P. Pekola, M. Manninen, Y.M. Galperin, Phys. Rev. B 70, 125425 (2004)

[18] T. Kühn, D.V. Anghel, Y.M. Galperin, M. Manninen, Phys. Rev. B 76, 165425 (2007), condmat/07051936

[19] B.A. Auld, Acoustic Fields and Waves in Solids, 2nd Ed. (Robert E. Krieger Publishing Company, 1990) 
